\title{
Island ordering on clean Pd(110)
}

\author{
H. Hörnis, J. R. West, and E. H. Conrad \\ School of Physics, Georgia Institute of Technology, Atlanta, Georgia 30332 \\ R. Ellialtioğlu \\ Physics Department, Bilkent University, Ankara, Turkey \\ (Received 1 February 1993; revised manuscript received 5 April 1993)
}

\begin{abstract}
High resolution low-energy electron diffraction measurements are reported that demonstrate the existence of semiordered islands on the clean $\mathrm{Pd}(110)$ surface. The islands are stable up to $1000^{\circ} \mathrm{C}$ and are approximately 90 atoms long in the $\langle 001\rangle$ direction. A simple model is presented that makes use of step-step interactions to generate the periodic island structure. This model predicts that ordered islands form below the roughening temperature if the step creation energy is small compared to the step-step interaction. A justification for this condition is given for the $\mathrm{Pd}(110)$ surface.
\end{abstract}

The formation of surface defects like steps, kinks, etc. have been actively studied over the past 10 years. ${ }^{1}$ These defects are present on miscut surfaces or on any surface above its roughening temperature $T_{R}\left(T_{R}\right.$ being the temperature where the free energy to form a step becomes zero). ${ }^{2}$ Since most models for roughening include only nearest-neighbor interactions, surfaces are predicted to be either rough or ordered. The addition of next-nearestneighbor interactions, however, allows new types of disordered phases to form below $T_{R}$. Examples include the next-nearest-neighbor-induced prerough phase of den $\mathrm{Nijs},{ }^{3}$ and the island structure caused by the asymmetry in the surface stress tensor between the $2 \times 1$ and $1 \times 2$ reconstructions on $\mathrm{Si}(001){ }^{4}$

In this work we demonstrate that over a wide temperature range, semiordered up-down steps are the lowestenergy surface configuration for the clean $\mathrm{Pd}(110)$ surface. This surface is therefore intermediate between an ordered phase and a rough phase. The islands formed by the step boundaries are found to be stable from room temperature up to $1000^{\circ} \mathrm{C}$ and are not associated with the sample miscut. We present a simple model that predicts that long-range step-step interactions can cause these islands to form if the energy to create a free step is small enough.

The experiments were performed using a high $Q$ resolution low-energy electron diffraction (LEED) system described elsewhere. ${ }^{5}$ The sample was a $99.995 \%$ pure Pd single crystal oriented to within $0.1^{\circ}$ of the nominal $\langle 110\rangle$ direction. After mechanical polishing, the sample was electropolished with a $50 \%$ sulfuric acid solution.

The Pd surface was cleaned in UHV by $\sim 1500$ cycles of argon-ion sputtering at $500 \mathrm{eV}$ for $10 \mathrm{~min}$ followed by annealing at $1000^{\circ} \mathrm{C}$ for $10 \mathrm{~min}$. After cleaning, Auger spectra showed no $\mathrm{C}, \mathrm{S}$, or $\mathrm{O}$ contamination down to the noise limit of the analyzer $(<1 \%$ of the $\mathrm{Pd} 320-\mathrm{eV}$ Auger line). This cleaning procedure allowed the sample to be heated at $1200^{\circ} \mathrm{C}$ for $30 \mathrm{~min}$ and then cooled to room temperature with no change in the diffraction intensity. The sample mosaic spread and finite domain size were found to be $\sim 0.04^{\circ}$ and $>1200 \AA$, respectively.

The main observation of this work is the appearance of strong satellite peaks near the specular rod for all sample temperatures below $1000^{\circ} \mathrm{C}$. This is shown in Fig. 1 . The data were taken by measuring the diffracted electron intensity as a function of their parallel momentum transfer vector $\left(Q_{\|}\right)$through the (110) point on the specular rod. All of the diffraction data is reported in the conventional bulk cubic reciprocal lattice units $a^{*}(h, k, l)$, where $a^{*}=1.615 \AA^{-1}$. The top panel in Fig. 1 is a scan in the $\langle 001\rangle$ direction (perpendicular to the atom rows: see inset in Fig. 1). Distinct shoulders corresponding to several orders of diffraction can be seen around the $Q_{\|}=0$ diffraction rod. The satellite peaks occur in integer spacings of $\Delta Q_{\|}=n 0.02 \pm 0.002 \AA^{-1}$, and both $n=1$ and 2 peaks can be resolved. Shoulders also appear in the

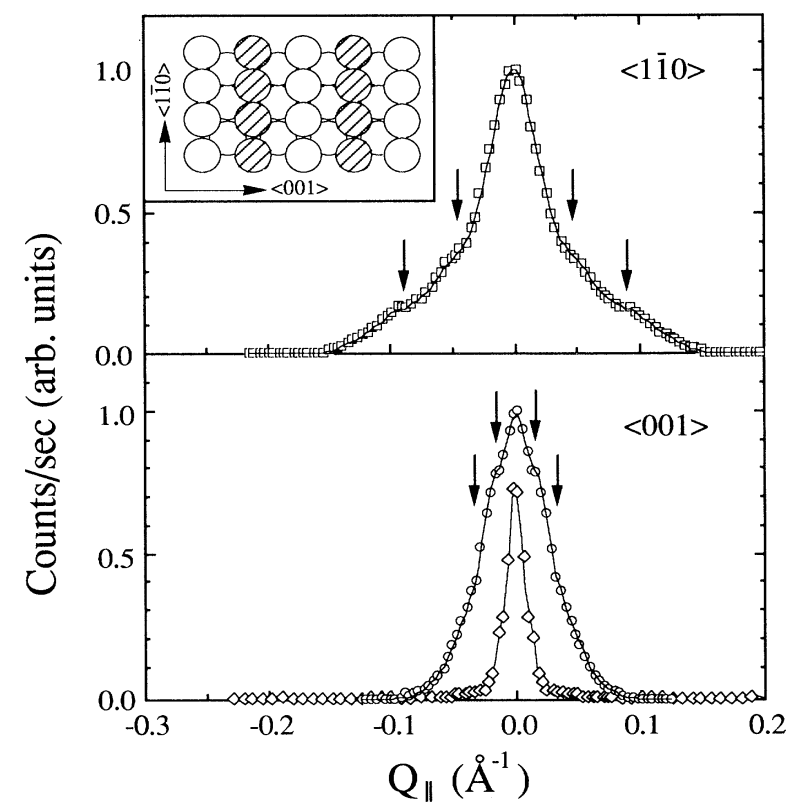

FIG. 1. $Q_{\|}$scans through the (110) point taken both perpendicular to the atom rows, $\langle 001\rangle(O)$ and along the rows, $\langle 1 \overline{1} 0\rangle$ $(\square)$. For comparison the $(220)$ in-phase peak is shown $(\diamond)$. The electron energy is $307 \mathrm{eV}$ and the incident angle (relative to the sample normal) is $82.7^{\circ}$. Arrows indicate the positions of the satellite peaks. Inset shows a top view of a (110) $1 \times 1 \mathrm{fcc}$ surface. Shaded atoms would be missing in a $2 \times 1$ structure. 
$\langle 1 \overline{1} 0\rangle$ direction as well (parallel to the atom rows in Fig. 1). In this direction the peak separations are larger, $\Delta Q_{\|}=n 0.050 \pm 0.002 \AA^{-1}$. It should be noted that the satellite peak separations are comparable or less than the $Q_{\|}$resolution of a typical LEED system $\left(\Delta Q_{\|}>0.04\right.$ $\AA^{-1}$ ). This explains why they have not been reported in the literature before now.

The satellite peaks are due to scattering from a surface superlattice structure with a periodicity of $\left(N_{1} \times N_{2}\right)$, where $N_{i}=2 \pi / a_{i} \Delta Q_{\| i}$. For $\operatorname{Pd}(110) a_{1}=3.89 \AA$ and $a_{2}=2.75 \AA$ in the $\langle 001\rangle$ and $\langle 1 \overline{1} 0\rangle$ directions, respectively. Using the experimental satellite spacings, $N_{1} \sim 80$ and $N_{2} \sim 46$. While it is difficult to determine how well ordered the superlattice is, the fact that the satellite peaks are resolvable implies that the structure repeats itself over several superlattice cells; $\sim 200 \AA$.

Within the experimental uncertainty $\left( \pm 0.004 \AA^{-1}\right)$, the satellite peak separation was found to be independent of sample temperature from $50^{\circ} \mathrm{C}$ up to $1000^{\circ} \mathrm{C}$ indicating that the surface structure responsible for the higher periodicity is very stable. A hydrogen-induced reconstruction can therefore be ruled out because the temperature stability of the structure is well above the hydrogen desorption temperature $\left(330^{\circ} \mathrm{C}\right){ }^{6}$ Although the periodicity of the structure is independent of temperature in this range, there is a temperature dependence of the distribution of atoms within the superlattice cell that will be discussed in a future paper. ${ }^{7}$

The question remaining to be answered is the following: what is the nature of the structure responsible for the higher-order periodicity? The satellite peaks cannot be due to the sample mosaic, since their angular separation is 5 times larger than the angular broadening measured at the $(220)$ position [a mosaic would produce the same angular broadening at both the $(110)$ and $(220)$ point $\left.{ }^{8}\right]$. The clue to the origin of the satellite peaks comes from their intensity variations as a function of $Q_{z}\left(Q_{z}\right.$ is the component of the electron momentum transfer perpendicular to the surface). The peaks only appear in a narrow range of $Q_{z}$ 's on the specular rod around the (110) point. For $Q_{z}=4.57 \AA^{-1}$ corresponding to the (220) point on the specular diffraction rod, no shoulders are seen (see Fig. 1).

These two observations indicate that the higher-order reconstruction is a result of ordered steps on the surface. This conclusion follows because the (110) point is an outof-phase diffraction condition ( $h, k$, and $l$ not all even or all odd). At an out-of-phase point steps cause the diffraction line shape to broaden (or add satellite peaks). ${ }^{8}$ On the other hand, the (220) position is an in-phase point ( $h, k$, and $l$ all even or all odd) and therefore insensitive to the presence of steps. Even when steps are present, the intensity and line shape of the (220) point remain unchanged from that expected for a flat surface. A finite sample domain size or faceting would broaden the (220) peak as well, which is clearly not observed (see Fig. 1). ${ }^{8}$

In order to estimate the structure of the superlattice we assume a simple one-dimensional model consisting of two levels separated by a monoatomic step. For convenience, the upper level will be referred to as the terrace and the lower level as the substrate. The repeat distance between terraces is $N a$ and the length of the upper terrace is $m a$ (where $N$ and $m$ are integers). The diffraction from this structure is a set of narrow reciprocal lattice rods with a separation of $\Delta Q_{\|}=2 \pi / N a$.

The peak intensities of the satellite rods depend on the relative length of the terraces compared to the length of the exposed substrate. In the case where $m=N / 2$, for example, there are an equal number of atoms in the terraces and the exposed substrate causing the specular intensity (at the out-of-phase condition) to be exactly zero. ${ }^{8}$ In this case the central peak would disappear leaving only the satellite peaks. When $m \neq N / 2$, the specular intensity is nonzero. Therefore, the ratio of the specular peak intensity to the satellite intensities gives the relative size of the upper and lower layers (i.e., this intensity ratio is a function of $[N-m] / m)$. To be more quantitative we write the scattered amplitude from the surface as

$$
\boldsymbol{A}(\boldsymbol{Q})=\sum_{p=0}^{M-1} e^{i p N a Q_{\|}}\left\{\sum_{n_{1}=0}^{m} e^{i n_{1} a Q_{\|}}+\sum_{n_{2}=0}^{N-m-1} \exp \left(i\left\{\left[n_{2}+\left(m+\frac{1}{2}\right)\right] a Q_{\|}-c Q_{z}\right\}\right)\right\}
$$

Squaring Eq. (1) gives the diffraction intensity $\left[I(Q)=A A^{*}\right]$. The ratio of the central peak intensity to the satellite peak intensity is found by calculating $I\left(Q_{\|}=0\right) / I\left(Q_{\|}=2 \pi n / N a\right)$, where $n$ is the order of the side peak.

By calculating $I\left(Q_{\|}=0\right) / I\left(Q_{\|}=2 \pi n / N a\right)$ from Eq. (1) for various values of $m$ and $N$ and comparing the results to the experimental intensity ratios, we find that the ratio of terrace atoms to substrate atoms is $2: 1$ for the $\langle 001\rangle$ direction and $3: 1$ for the $\langle 1 \overline{1} 0\rangle$ direction. Note that the surface could equally be described as having terrace to substrate ratios of $1: 2$ and $1: 3$ as well. The diffraction data do not allow us to determine whether there are more or less atoms in the terraces compared to the substrateit can only determine the ratio.

Note that this calculation ignores any distribution of terrace sizes. The fact that satellite peaks are observed, however, indicates that the distribution of terrace sizes must be peaked around some average size implying some type of step-step interaction. In contrast, a noninteracting step model would produce a geometric distribution of terrace sizes resulting in a two component line shape consisting of a sharp central peak centered on top of a broad Lorentzian background. ${ }^{9}$ In other words a noninteracting step model would not predict satellite peaks. From the measured satellite peak widths, we find that the island width distribution is about $20 \%$ broader in the $\langle 1 \overline{1} 0\rangle$ direction.

To summarize, we propose that the low-temperature surface of $\mathrm{Pd}(110)$ consists of a semiperiodic array of islands. The island sizes in the $\langle 1 \overline{1} 0\rangle$ direction are about $30 \%$ of the sizes in the $\langle 001\rangle$ direction. The smaller is- 


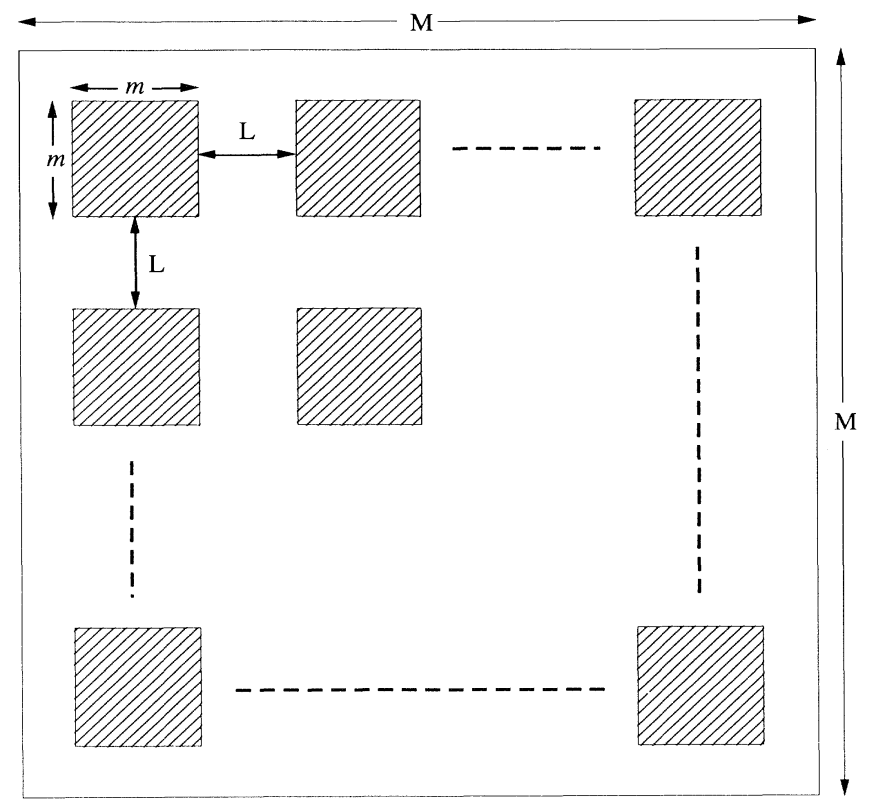

FIG. 2. A schematic representation of the model Pd(110) surface described in the text. The shaded squares are terraces one atomic step high.

land size in the $\langle 1 \overline{1} 0\rangle$ direction is most likely due to fluctuations of the $\langle 001\rangle$ step edges (i.e., step edge roughness). In the $\langle 001\rangle$ direction the mean length of an island $(N-m)$ is about $26 \pm 5$ atoms and the mean separation between islands $(m)$ is about $54 \pm 5$ atoms. The corresponding dimensions in the $\langle 1 \overline{1} 0\rangle$ direction are $N-m=11 \pm 5$ and $m=35 \pm 2$ atoms.

We now propose a model that can rationalize the existence of the observed island structure on this surface. Consider an ordered array of $p \times p$ square islands on a flat substrate $M \times M$ atoms wide (see Fig. 2). Each island contains $m \times m$ atoms. All islands are equally spaced with a separation distance $L a$ (where $L$ is an integer and $M=p[m+L])$. Ignoring the crystallographic anisotropy for simplicity, the free energy difference between an island covered surface and a flat surface without islands will be

$$
\Delta F=\gamma 4 m p^{2}+m p^{2} \sigma\left(1 / L^{2}+1 / m^{2}\right) .
$$

The first term in Eq. (2) is the step free energy per unit length of the island perimeter $\gamma$. The second term is the step-step interaction energy that has contributions from inter- and intra-island step edges. The step-step interaction is presumed to be elastic (rather than entropic) in origin. ${ }^{10}$ Contributions to Eq. (2) that arise from the configurational entropy of the islands are neglected in this simple treatment.

This model predicts that $\Delta F$ will always have a minimum for some combination of $L$ and $m$ if $\gamma<0$ and the steps are repulsive. In other words, ordered steps are preferred over the flat surface. The model also predicts that while the length of a terrace is a function of $\gamma / \sigma$, the terrace-substrate ratio is almost independent of $\gamma / \sigma$ (a result of the $1 / x^{2}$ dependence of the step-step interaction). This may explain why the experimentally determined ratios in the two orthogonal directions are almost the same in spite of the asymmetry in the Pd(110) surface geometry.

Without step-step interactions, the condition that the step free energy is less than zero implies that the surface is above its roughening temperature and that ordered islands cannot exist. ${ }^{2}$ But we argue that a long-range step repulsion renormalizes the step free energy and has the effect of raising the roughening temperature so that ordered steps can still exist at finite temperatures. This is completely analogous to the situation of vicinal metal surfaces where an ordered step staircase exists because of step-step interactions. ${ }^{11}$ On these surfaces $T_{R}$ increases as the step-step interaction increases (i.e., as the terrace length between steps decreases). ${ }^{1}$

Whether or not the structure we propose is favorable depends crucially on $\operatorname{Pd}(110)$ having a small step energy. The (110) fcc metals are rather unique because some of them ( $\mathrm{Au}, \mathrm{Pt}, \mathrm{Ir})$ have a $2 \times 1$ missing-row reconstruction. ${ }^{12}$ The nature of this reconstruction is important to this work. The (110) $2 \times 1$ surface is essentially an ordered arrangement of steps (see inset in Fig. 2). The energy difference $\Delta E_{2 \times 1}$ between the $2 \times 1$ and $1 \times 1$ surface is therefore closely related to the energy cost to produce a step. The reconstruction energy has been calculated by several groups using embedded-atom potentials. ${ }^{13}$ It is found that $\Delta E_{2 \times 1}$ for $\mathrm{Pd}$ and $\operatorname{Ag}(110)$ is either small or negative indicating that the cost of making a step on these surfaces is low (at least as predicted by these models). So it seems reasonable to assume that $\gamma$ for $\operatorname{Pd}(110)$ may indeed be small.

We note that den Nijs suggested that if the energy to make a step is low on a (110) surface and that nextnearest-neighbor interactions are strong enough, the surface can become "prerough." 3 The prerough phase is flat (a finite height-height correlation function) with no longrange order, but does consist of a series of correlated updown steps. That is, every up step is followed by a down step. Even though the model we present for $\operatorname{Pd}(110)$ is a "flat" phase, some long-range order still remains. Indeed on Pd, and probably on most metal surfaces, step-step interactions may prevent a true prerough phase from forming.

We wish to thank Professor A. Zangwill for suggesting the possible importance of step-step interactions to this problem. This work has been supported by the NSF under Grant No. DMR-9211249 and by a NATO travel grant. 


\section{RAPID COMMUNICATIONS}

${ }^{1}$ For a review see, Edward H. Conrad, Prog. Surf. Sci. 39, 65 (1992).

${ }^{2} \mathrm{H}$. van Beijeren and I. Nolden, in Structures and Dynamics of Surfaces, edited by W. Schommers and P. von Blanckenhagen (Springer, Heidelberg, 1987).

${ }^{3}$ M. den Nijs, Phys. Rev. Lett. 64, 435 (1990).

${ }^{4}$ O. L. Alerhand, D. Vanderbilt, R. D. Meade, and J. D. Joannopoulos, Phys. Rev. Lett. 61, 1973 (1988): F. K. Men, W. E. Packard, and M. B. Webb, ibid. 61, 2469 (1988).

${ }^{5}$ Y. Cao and E. H. Conrad, Rev. Sci. Instrum. 60, 2642 (1989).

${ }^{6}$ R. J. Behm, K. Christmann, and G. Ertl, Surf. Sci. 99, 320 (1980).

${ }^{7}$ H. Hörnis, J. R. West, E. H. Conrad, and R. Ellialtioğlu (unpublished).

${ }^{8}$ M. Henzler, in Electron Spectroscopy for Surface Analysis, edited by H. Ibach (Springer, Berlin, 1979).
${ }^{9}$ C. S. Lent and P. I. Cohen, Surf. Sci. 139, 121 (1984).

${ }^{10}$ A. F. Andreev and Yu. A. Kosevich, Zh. Eksp. Teor. Fiz. 81, 1435 (1981) [Sov. Phys. JETP 54, 761 (1981)].

${ }^{11}$ J. Villain, D. R. Grempel, and J. Lapujoulade, J. Phys. F 15, 809 (1985).

${ }^{12}$ W. Moritz and D. Wolf, Surf. Sci. 163, L655 (1985); I. K. Robinson, Phys. Rev. Lett. 50, 1145 (1983); L. D. Marks, ibid. 51, 1000 (1983); M. Copel and T. Gustafson, ibid. 57, 723 (1986); E. C. Sowa, M. A. van Hove, and D. L. Adams, Surf. Sci. 199, 174 (1988); G. L. Kellog, Phys. Rev. Lett. 55, 2168 (1985); P. Ferry, W. Moritz, and D. Wolf, Phys. Rev. B 38, 7275 (1988); P. Ferry, W. Moritz, and D. Wolf, ibid. 38, 7275 (1988), and references therein.

${ }^{13}$ S. M. Foiles, Surf. Sci. 191, L779 (1987); S. P. Chen and A. F. Voter, Surf. Sci. Lett. 244, L107 (1991). 